\title{
Rapamycin protects dopaminergic neurons against rotenone- induced cell death in primary mesencephalic cell culture
}

\author{
Khaled Radad ${ }^{1}$, Rudolf Moldzio², Wolf-Dieter Rausch ${ }^{2}$ \\ ${ }^{1}$ Department of Pathology, Faculty of Veterinary Medicine, Assiut University, Egypt, ${ }^{2}$ Institute of Medical Biochemistry, Department \\ for Biomedical Sciences, University of Veterinary Medicine, Vienna, Austria
}

\begin{abstract}
Introduction: Parkinson's disease is the most common movement disorder, characterized by a progressive and extensive loss of dopaminergic neurons in the substantia nigra pars compacta and their terminals in the striatum. So far, only symptomatic treatment is available, and no cure or disease-modifying drugs exist. The present study was designed to investigate the neuroprotective effect of rapamycin, an autophagy inducer, on dopaminergic neurons against rotenone-induced cell death in primary mesencephalic cell culture.

Material and methods: Primary mesencephalic cell cultures were prepared from embryonic mouse mesencephala (OFI/SPF, Vienna, Austria) at gestation day 14. Four sets of cultures were treated as follows: one was run as an untreated control, a second one was treated with $20 \mathrm{nM}$ rotenone on the $10^{\text {th }}$ day in vitro (DIV) for $48 \mathrm{~h}$, a third one was co-treated with $20 \mathrm{nM}$ rotenone and rapamycin $(1,10,100,1000 \mathrm{nM})$ on the $10^{\text {th }} \mathrm{DIV}$ for $48 \mathrm{~h}$, and a fourth one was treated with rapamycin alone $(1,10,100,1000 \mathrm{nM})$ on the $10^{\text {th }}$ DIV for $48 \mathrm{~h}$. On the $12^{\text {th }} \mathrm{DIV}$, cultures were subjected to immunohistochemistry against tyrosine hydroxylase and to fluorescence staining using LysoTracker Deep Red, $J C-1$ and DAPI stains.

Results: Exposure of such cultures to $20 \mathrm{nM}$ rotenone on the $10^{\text {th }} \mathrm{DIV}$ for $48 \mathrm{~h}$ reduced the number of dopaminergic neurons by $41 \%$ and increased the release of lactate dehydrogenase (LDH) by $178 \%$ above untreated controls. Rapamycin $(1,10,100,1000 \mathrm{nM})$ added together with rotenone from the $10^{\text {th }}$ to $12^{\text {th }}$ DIV spared dopaminergic neurons by $17 \%$ and reduced the release of $L D H$ by $64 \%$ at the concentration of $100 \mathrm{nM}$ compared to rotenone-treated cultures. Activation of an autophagic process by rapamycin was demonstrated by LysoTracker Deep Red fluorescent dye, as indicated by a shift to increased red fluorescence. Rapamycin also significantly elevated the mitochondrial membrane potential $(\Delta \psi \mathrm{m})$, as shown by an increase of the red:green fluorescence ratio of $J C-1$. Increased apoptotic cell death due to rotenone was lowered by rapamycin, as shown by the blue-fluorescent DAPI nucleic acid stain.

Conclusions: Our study indicates for the first time that rapamycin, known as an autophagy inducer, protected dopaminergic neurons against rotenone-induced cell death in primary mesencephalic cell culture.
\end{abstract}

Key words: apoptosis, autophagy, dopaminergic neurons, neuroprotection, Parkinson's disease, rapamycin. 


\section{Introduction}

Parkinson's disease (PD) is a common progressive neurodegenerative disorder affecting more than 6 million people worldwide [28]. The disease is characterized clinically by a triad of cardinal motor symptoms including bradykinesia, tremors and rigidity [13], and pathologically by the selective loss of dopamine neurons in the substantia nigra pars compacta (SNpc) and the formation of Lewy bodies [27]. Although the etiology of PD is still unclear, there is growing evidence indicating that intracellularly oxidative stress, mitochondrial damage, lysosomal dysfunction, neuroinflammatory changes and formation of pathologic inclusions contribute to the pathology of the disease [9].

Treatment of PD is generally symptomatic, where levodopa remains the most effective agent [34]. However with disease progression, levodopa medication becomes increasingly inadequate for the management of motor fluctuations and dyskinesias [40]. Levodopa given clinically undergoes autooxidation and forms reactive oxygen species (ROS) which could be toxic to remaining dopamine neurons [20]. Dopamine agonists and monoamine oxidase B inhibitors are also prescribed for the treatment of early PD as in addition to their symptomatic benefits they postpone the onset of levodopa therapy [11].

During the last two decades, researchers have shown increased interest in developing neuroprotective substances that can slow or stop the clinical progression of PD. As a result, significant numbers of compounds have been identified as neuroprotective in preclinical studies [8]. For instance, the D3/ D2/D1 dopamine receptor agonist rotigotine was shown to protect dopaminergic neurons against glutamate, $\mathrm{MPP}^{+}$and rotenone in primary mesencephalic cell culture $[23,32]$. The active principal of Nigella sativa seed thymoquinone protected dopaminergic neurons against $\mathrm{MPP}^{+}$-induced cell death in primary mesencephalic cell culture [30]. Inhibition of sphingosine kinase (Sphk1), a regulator of bioactive sphingolipid homeostasis, by SKI II protected the dopaminergic SH-SY5Y cells through an antiapoptotic pathway [29]. However, to date, there is no drug that has provided neuroprotection against dopaminergic cell loss in clinical trials [4].

Rapamycin is a lipophilic macrocyclic antibiotic produced by the bacterium Streptomyces hygroscopicus indigenous to Easter Island [38]. Rapamycin was shown first to exhibit antibacterial, antifungal, immunosuppressive and anticancer effects [33]. Recently, rapamycin has been reported to reduce cytotoxic injury in different models of neurodegenerative disorders [5]. For instance, Park et al. [26] reported that rapamycin protected human neuroblastoma SH-SY5Y cells against fipronil-induced apoptotic cell death. Kanno et al. [18] found that rapamycin reduced locomotor impairment and neuronal death after spinal cord injury in mice. This effect of rapamycin was reported to be mediated through activation of autophagy by inhibiting the mammalian target of rapamycin (mTOR) signaling pathways [36].

More recently, studies using post-mortem human tissues and genetic and toxin-induced animal and cellular models have implicated autophagy dysfunction as an important issue in PD pathogenesis [1]. For instance, Dehay et al. [7] reported that the number of undegraded autophagosomes increased and the number of autophagolysosomes decreased in post-mortem PD brain samples. Park et al. [25] showed that MPP+ inhibited autophagosome formation and increased $\alpha$-synuclein expression in mice. Parganlija et al. [24] found that SH-SY5Y cells with PINK1 knockdown showed down-regulation of key autophagic genes including Beclin, LC3 and LAMP-2. Accordingly, our present study was designed to investigate the neuroprotective potential of the autophagy inducer rapamycin against rotenone-induced dopaminergic cell death relevant to PD. To date, there have been no reports describing the potential neuroprotective role of rapamycin on dopamine neurons in primary mesencephalic cell culture relevant to $\mathrm{PD}$.

\section{Material and methods \\ Preparation of primary mesencephalic cell culture}

Primary mesencephalic cell cultures were prepared from OFI/SPF embryos according to Meinel et al. [22]. In brief, embryonic mouse mesencephala were dissected on the $14^{\text {th }}$ day of gestation and cut into small pieces in a drop of DPBS (Invitrogen, Germany), $2 \mathrm{ml}$ of $0.2 \%$ trypsin solution (Invitrogen, Germany) and $2 \mathrm{ml}$ of $0.02 \%$ DNase I solution (Roche, Germany) were added and the tissue was subsequently incubated in a water bath at $37^{\circ} \mathrm{C}$ for $7 \mathrm{~min}$. Then, $2 \mathrm{ml}$ of trypsin inhibitor $(0.125 \mathrm{mg} / \mathrm{ml})$ (Invitrogen, Ger- 
many) were added, the tissue was centrifuged at $100 \times \mathrm{g}$ for $4 \mathrm{~min}$ and the supernatant was aspirated. The tissue pellet was triturated 2-3 times with a fire-polished Pasteur pipette; each time 0.02\% DNase I (Invitrogen, Germany) was included in the medium. Dissociated cells were plated at a density of 257000 cells $/ \mathrm{cm}^{2}$ in DMEM (Sigma, Germany) supplemented with $4 \mathrm{mM}$ glutamine, $10 \mathrm{mM}$ HEPES buffer, $30 \mathrm{mM}$ glucose, $100 \mathrm{IU} / \mathrm{ml}$ penicillin, $0.1 \mathrm{mg} / \mathrm{ml}$ streptomycin and 10\% heat-inactivated fetal calf serum (Sigma, Germany). The medium was exchanged on the $1^{\text {st }}$ day in vitro (DIV) and on the $3^{\text {rd }}$ DIV. On the $5^{\text {th }}$ DIV half of the medium was replaced with serum-free DMEM containing $0.02 \mathrm{ml}$ of B-27/ml (Invitrogen, Germany) DMEM. Serum-free supplemented DMEM was used for feeding from the $6^{\text {th }}$ DIV and subsequently replaced every $2^{\text {nd }}$ day.

\section{Treatment of cultures with rapamycin}

A stock solution of $1 \mathrm{mM}$ rapamycin (Invitrogen, USA) was prepared in dimethyl sulfoxide (DMSO) and further diluted in DMEM to final concentrations. On the $10^{\text {th }} \mathrm{DIV}$, cultures were treated with rapamycin $(1,10,100,1000 \mathrm{nM})$ for $48 \mathrm{~h}$ to investigate its effect on the survival of dopaminergic cells.

\section{Treatment of cultures with rapamycin and rotenone}

A stock solution of $1 \mu \mathrm{M}$ of rotenone (Sigma-Aldrich, Germany) was prepared in DMSO and then diluted in DMEM to final concentrations. For each treatment, fresh rotenone solutions were used to avoid breakdown of rotenone by storage. To investigate the neuroprotective potential of rapamycin against rotenone-induced dopaminergic cell death, cultures were co-administered with both rotenone (20 nM) and rapamycin (1, 10, 100, $1000 \mathrm{nM})$ on the $10^{\text {th }}$ DIV for $48 \mathrm{~h}$.

\section{Identification of dopaminergic neurons}

Dopaminergic neurons were identified immunocytochemically by tyrosine hydroxylase staining. On the $12^{\text {th }}$ DIV, cultures were rinsed carefully with phosphate buffered saline (PBS, $\mathrm{pH} 7.2$ ) and fixed in $4 \%$ paraformaldehyde for $45 \mathrm{~min}$ at $4^{\circ} \mathrm{C}$. After washing with PBS, cells were permeabilized with $0.4 \%$ Triton X-100 for $30 \mathrm{~min}$ at room temperature. Cultures were washed 3 times with PBS and incubated with $5 \%$ horse serum (Vectastain ABC Elite kit) for 90 min to block nonspecific binding sites. To determine the number of tyrosine hydroxylase immunoreactive (THir) cells, cultures were sequentially incubated with anti-TH primary antibody overnight at $4{ }^{\circ} \mathrm{C}$, biotinylated secondary antibody (Vectastain) and avidin-biotin-horseradish peroxidase complex (Vectastain) for 90 min at room temperature and washed with PBS between stages. The reaction product was developed in a solution of diaminobenzidine (1.4 mM) in PBS containing $3.3 \mathrm{mM}$ hydrogen peroxide $\left(\mathrm{H}_{2} \mathrm{O}_{2}\right)$, and stained cells were counted with a Nikon inverted microscope in 10 randomly selected fields per well at $10 \times$ magnification.

\section{Measurement of LDH activity}

Cellular injury was quantitatively assessed by measuring the activity of lactate dehydrogenase $(\mathrm{LDH})$ released from damaged cells into the culture medium. The reaction was initiated by mixing $0.2 \mathrm{ml}$ of cell-free supernatant (diluted $1: 1$ with distilled water) with potassium phosphate buffer containing $\beta$-nicotinamide adenine dinucleotide (NADH) and sodium pyruvate (0.18 and $0.62 \mathrm{mM}$ in potassium phosphate buffer, respectively) in a final volume of $0.5 \mathrm{ml}$ in $1 \mathrm{ml}$ cuvettes. The decrease of NADH was spectrophotometrically (NOVASPEC1 II) monitored. Reagent blanks were subtracted. LDH activity was calculated from the slope of the decrease in optical density at $334 \mathrm{~nm}$ over a $3 \mathrm{~min}$ time period. The LDH release is proportional to the number of damaged or destroyed cells $[10,19]$.

\section{Staining of cultured cells with LysoTracker Deep Red fluorescent dye}

LysoTracker dye is a highly soluble small molecule that is retained in acidic subcellular organelles such as lysosomes. It is used to investigate the biosynthesis of lysosomes. Here, $100 \mathrm{nM}$ rapamycin (the concentration significantly protected dopaminergic neurons in rotenone-treated cultures) was added together with $20 \mathrm{nM}$ rotenone on the $10^{\text {th }}$ DIV for $48 \mathrm{~h}$. On the $12^{\text {th }}$ DIV, culture media were aspirated and cells were incubated with a new medium containing 100 nM LysoTracker Deep Red fluorescent dye (Life Technologies, Invitrogen, USA) for 15-30 min at $37^{\circ} \mathrm{C}$. After washing with DPBS, stained cells were photographed on a Nikon inverted microscope equipped with an epifluorescence attachment using a rhodamine filter set with an excitation wavelength 
of 580 and an emission wavelength of 590, G-2A and a Coolpix 990 digital camera (Nikon, Japan). Six photos were taken randomly from each well (24 photos per experiment). All photos were analyzed densitometrically using Adobe Photoshop software.

\section{Measurement of $\Delta \psi \mathrm{m}$ by JC-1 fluorescent dye}

JC-1 is a lipophilic cationic dye that selectively enters mitochondria. In healthy cells with high mitochondrial $\Delta \psi \mathrm{m}, \mathrm{JC}-1$ spontaneously forms complexes known as J-aggregates with intense red fluorescence. In the case of apoptotic cells the dye remains in its monomeric form with green fluorescence. The JC-1 red:green ratio has been used as a tool to estimate changes in $\Delta \psi \mathrm{m}$ [39]. JC-1 was dissolved in DMSO and further diluted in DMEM $(10 \mu \mathrm{g} / \mathrm{ml}$ final concentration). After removal of the culture medium cells were loaded with JC-1 for $15 \mathrm{~min}$ at $37^{\circ} \mathrm{C}$, rinsed twice with PBS and photographed on a Nikon inverted microscope equipped with an epifluorescence attachment using a rhodamine filter set with an excitation wavelength of $510 \mathrm{DM}$ and an emission wavelength of 520 BA and a Coolpix 990 digital camera (Nikon, Japan). Six photos were taken randomly from each well (24 photos per experiment). Fluorescence intensity of the red:green ratio was determined semiquantitatively using Adobe Photoshop software.

\section{Counting of apoptotic cells by blue- fluorescent DAPI nucleic acid stain}

DAPI is a fluorescent stain that binds strongly to DNA. It passes through intact membranes of live and fixed cells. Cells were fixed with $4 \%$ paraformaldehyde for $45 \mathrm{~min}$ at $4^{\circ} \mathrm{C}$. After washing with PBS $(\mathrm{pH} 7.2)$, cells were permeabilized with $0.4 \%$ Triton $\mathrm{X}-100$ for $30 \mathrm{~min}$ at room temperature. DAPI solution ( $2 \mu \mathrm{M}$ final concentration) was added to the cultures at room temperature for $5 \mathrm{~min}$ in the dark. After washing with DPBS, six photos were taken randomly from each well (24 photos per experiment) with a Coolpix 990 digital camera connected to an inverted microscope with an epifluorescence attachment using an ultraviolet (UV) filter (Nikon, Japan). Nuclei with condensed and fragmented chromatin were counted when the photos were reviewed with Adobe Photoshop software.

\section{Statistics}

Each experiment was run in triplicate with four wells in each treatment. Data were expressed as mean \pm standard error of mean (SEM). Comparisons were made using ANOVA and post-hoc Duncan's test using the statistical program SAS 1998. $P<0.05$ was considered as statistically significant.

\section{Results}

\section{Rapamycin did not affect the survival of dopaminergic neurons}

Treatment of cultures with rapamycin $(1,10,100$, $1000 \mathrm{nM}$ ) on the $10^{\text {th }}$ DIV for $48 \mathrm{~h}$ did not affect the survival (Fig. 1) or the morphology of dopaminergic neurons (data not shown).

\section{Rapamycin rescued dopaminergic neurons from rotenone-induced cell death}

Treatment of cultures with $20 \mathrm{nM}$ rotenone on the $10^{\text {th }}$ DIV for $48 \mathrm{~h}$ decreased the number of dopaminergic neurons by $41 \%$ and altered the morphology of surviving neurons compared to untreated controls (Fig. 2A,B). On the other hand, co-administration of rapamycin and rotenone on the $10^{\text {th }}$ DIV for $48 \mathrm{~h}$ significantly increased the survival of dopaminergic

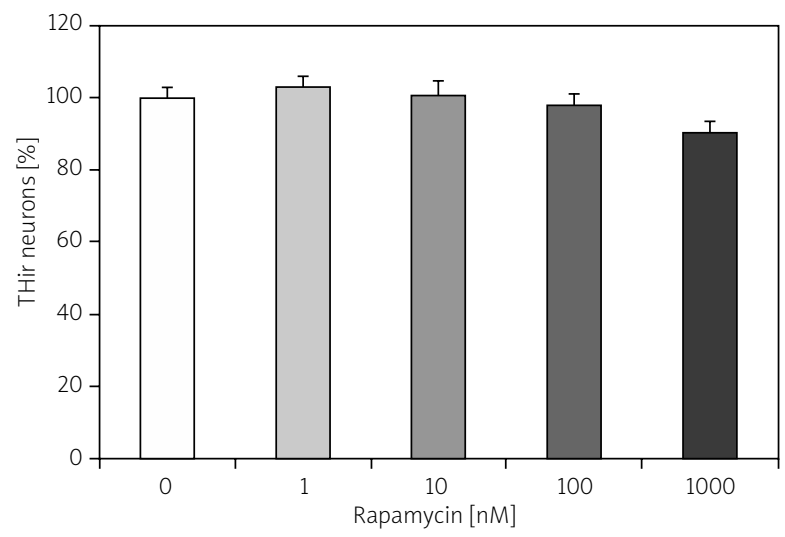

Fig. 1. Treatment of primary mesencephalic cell cultures with rapamycin on the $10^{\text {th }}$ DIV for 48 h. $100 \%$ corresponds to the total number of THir neurons (the average number of THir neurons was 26 cells/field) after 12 DIV in untreated controls. Values represent the mean \pm SEM of three independent experiments with four wells in each treatment. In each well THir neurons were counted in ten randomly selected fields. 


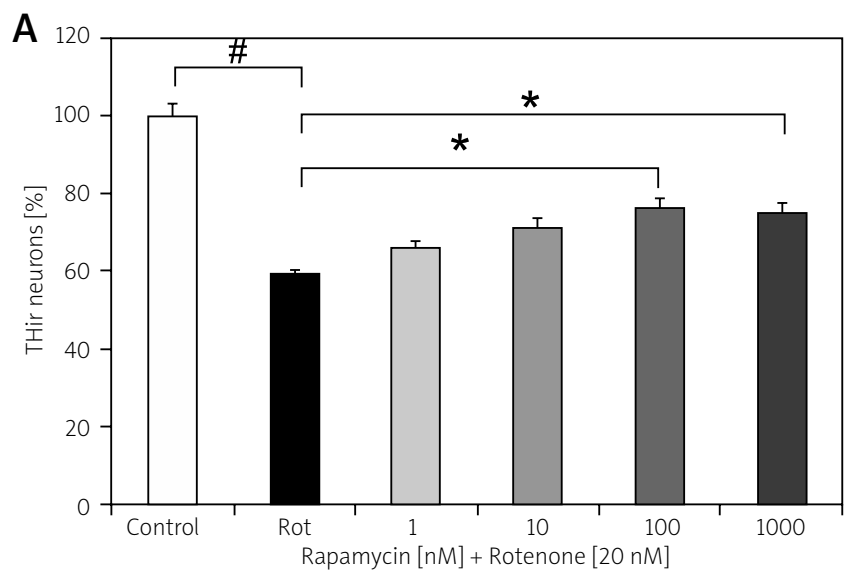

B
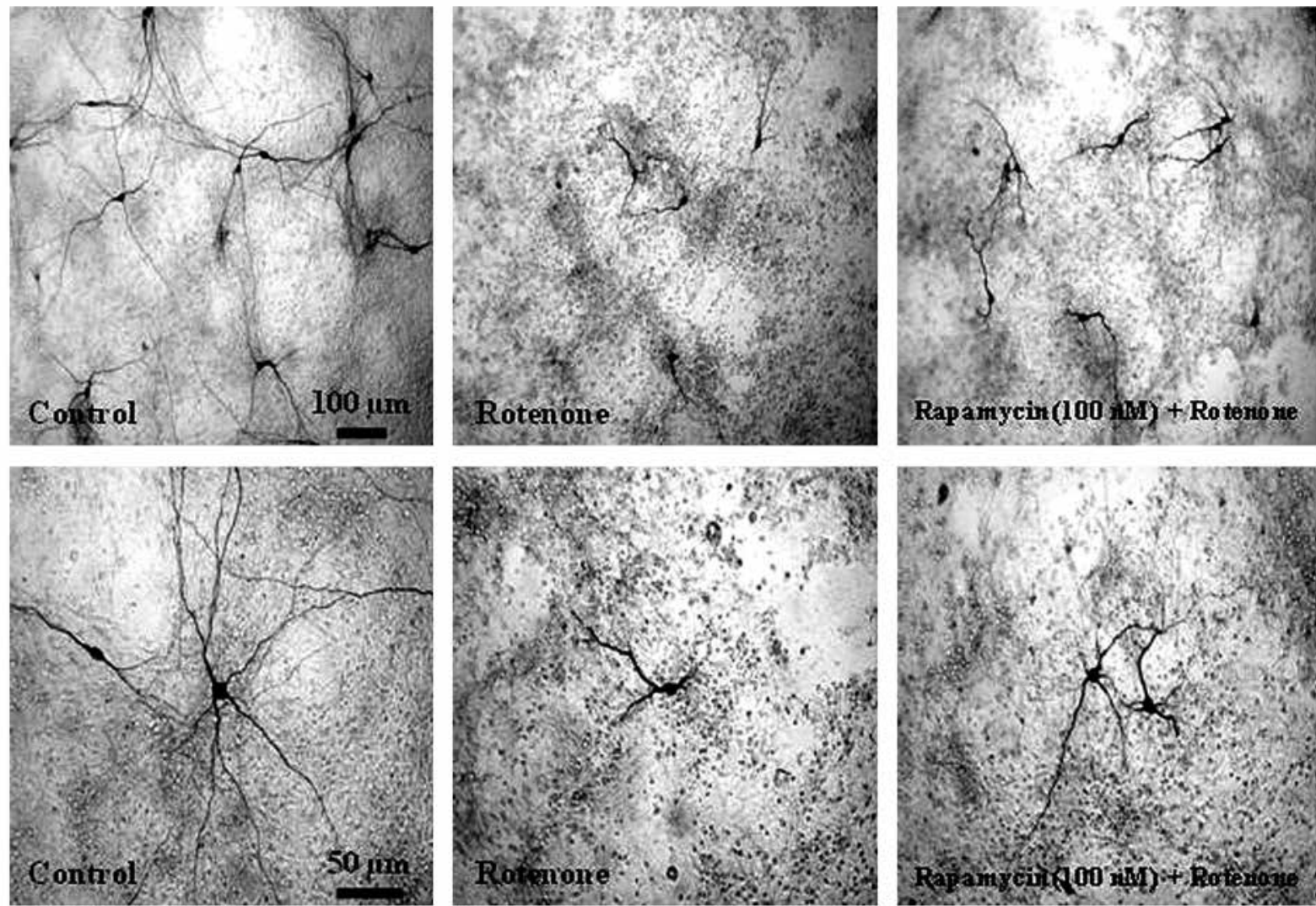

Fig. 2. A) Concomitant treatment of primary mesencephalic cell cultures with rotenone $(20 \mathrm{nM})$ and rapamycin $(1,10,100,1000 \mathrm{nM})$ on the $10^{\text {th }}$ DIV for $48 \mathrm{~h}$. 100\% corresponds to the total number of THir neurons (the average number of THir neurons was 23 cells/field) after 12 DIV in untreated controls. Values represent the mean \pm SEM of three independent experiments with four wells in each treatment. In each well THir neurons were counted in ten randomly selected fields ( $\left.{ }^{\#} p<0.001,{ }^{*} p<0.001\right)$. B) Representative micrographs of THir neurons after 12 DIV. Untreated control cultures showed THir neurons with long and branched processes. Rotenone-treated cultures showed THir neurons with very few, shortened and thickened neurites. Treatment with rapamycin improves the morphology of THir neurons compared to rotenone-treated cultures. 
A

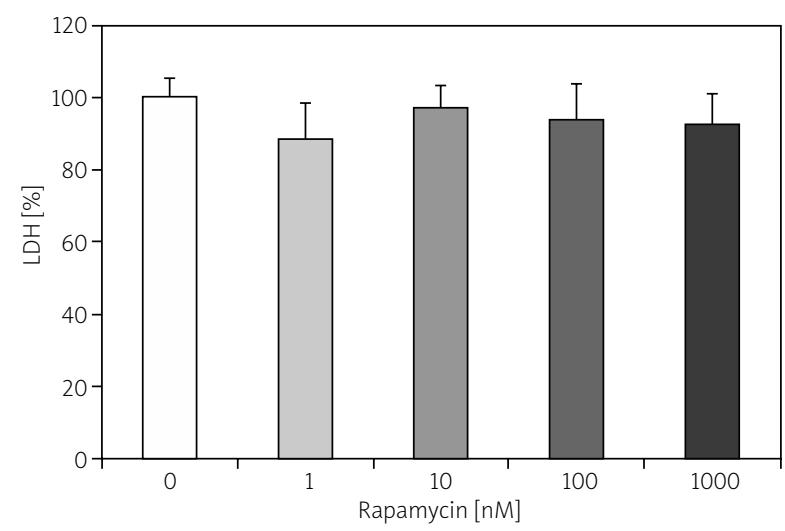

B

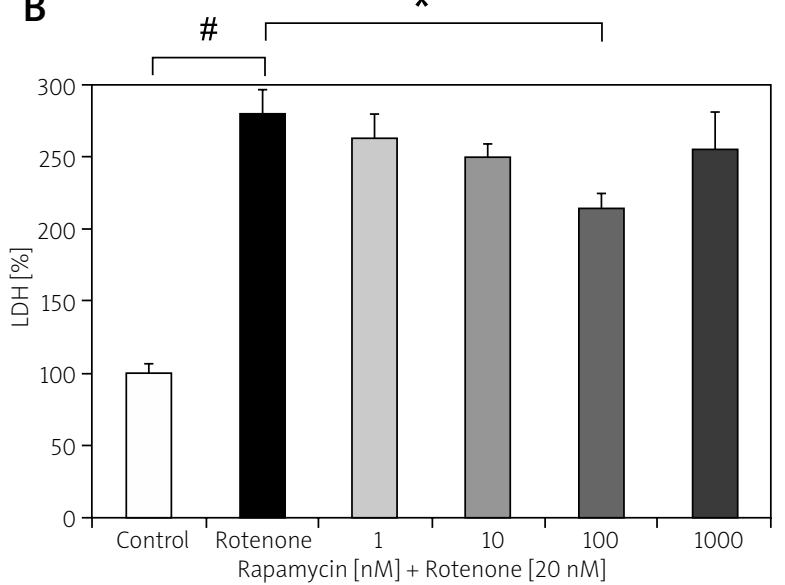

Fig. 3. Release of LDH into the culture medium in primary mesencephalic cell cultures. A) Treatment of cultures with rapamycin on the $10^{\text {th }}$ DIV for $48 \mathrm{~h}$. B) Concomitant treatment of cultures with rotenone $(20 \mathrm{nM})$ and rapamycin $(1,10,100,1000 \mathrm{nM})$ on the $10^{\text {th }}$ DIV for $48 \mathrm{~h} .100 \%$ corresponds to the amount of $\mathrm{LDH}$ in the culture medium after $12 \mathrm{DIV}$. Values represent the mean \pm SEM of three independent experiments with four wells in each treatment $\left({ }^{*} p<0.001,{ }^{*} p<0.001\right)$.

neurons by $17 \%$ compared to rotenone-treated cultures (Fig. 2A). Also, rapamycin reduced degenerative changes in dopaminergic neurons seen with rotenone exposure (Fig. 2B).

\section{Rapamycin attenuated rotenone- induced $\mathrm{LDH}$ release}

Treatment of cultures with rapamycin on the $10^{\text {th }}$ DIV for $48 \mathrm{~h}$ did not affect the level of LDH in the culture medium (Fig. 3A). Consistent with the cytotoxic effect of rotenone on dopaminergic neurons, $20 \mathrm{nM}$ rotenone led to an increase in release of $L D H$ into the culture medium by $178 \%$ compared to untreated controls (Fig. 4B). Against rotenone, rapamycin (100 nM) reduced the release of $\mathrm{LDH}$ by $64 \%$ compared to rotenone-treated cultures (Fig. 3B).

\section{Rapamycin increased LysoTracker Deep Red fluorescence}

Co-treatment of cultures with rapamycin (100 nM) and rotenone $(20 \mathrm{nM})$ on the $10^{\text {th }}$ DIV for $48 \mathrm{~h}$ significantly increased the fluorescent intensity of LysoTracker Deep Red by about 63\% compared to rotenone-treated cultures (Fig. 4A). In parallel, Fig. 4B shows higher red fluorescence in the cultures co-treated with rapamycin and rotenone compared to cultures treated with rotenone alone. Rapamycin alone did not produce a significant increase in the fluorescent intensity of LysoTracker Deep Red compared to untreated control culture (Fig. 4A,B).

\section{Rapamycin increased red: green fluorescence ratio of JC-1}

Treatment of cultures with rotenone $(20 \mathrm{nM})$ on the $10^{\text {th }}$ DIV for $48 \mathrm{~h}$ decreased the red:green fluorescent ratio of JC- 1 by about $29 \%$ compared to untreated controls (Fig. 5A). On the other hand, concomitant treatment of cultures with $100 \mathrm{nM}$ rapamycin and $20 \mathrm{nM}$ rotenone on the $10^{\text {th }}$ DIV for 48 $\mathrm{h}$ significantly restored the red:green fluorescent ratio of JC-1 by $19 \%$ compared to rotenone-treated cultures (Fig. 5A). Figure 5B showed that cultures co-administered with rapamycin and rotenone displayed much higher red fluorescence than the cultures treated with rotenone alone. Rapamycin alone did not significantly affect the red:green fluorescent ratio of JC compared to untreated control culture (Fig. 5A,B).

\section{Rapamycin decreased rotenone- induced apoptotic cell death}

Staining of cultured cells with the nuclear fluorescence dye DAPI revealed that rotenone $(20 \mathrm{nM}$ on the $10^{\text {th }}$ DIV for $48 \mathrm{~h}$ ) increased the number of nuclei showing apoptotic features by $119 \%$ compared to untreated cultures (Fig. 6A). Against rotenone, rapamycin was found to decrease the number of apoptotic 


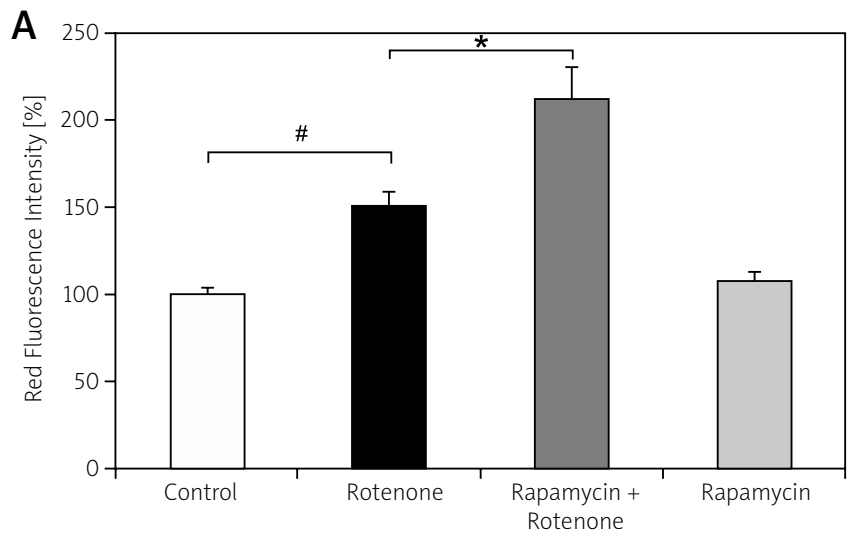

B
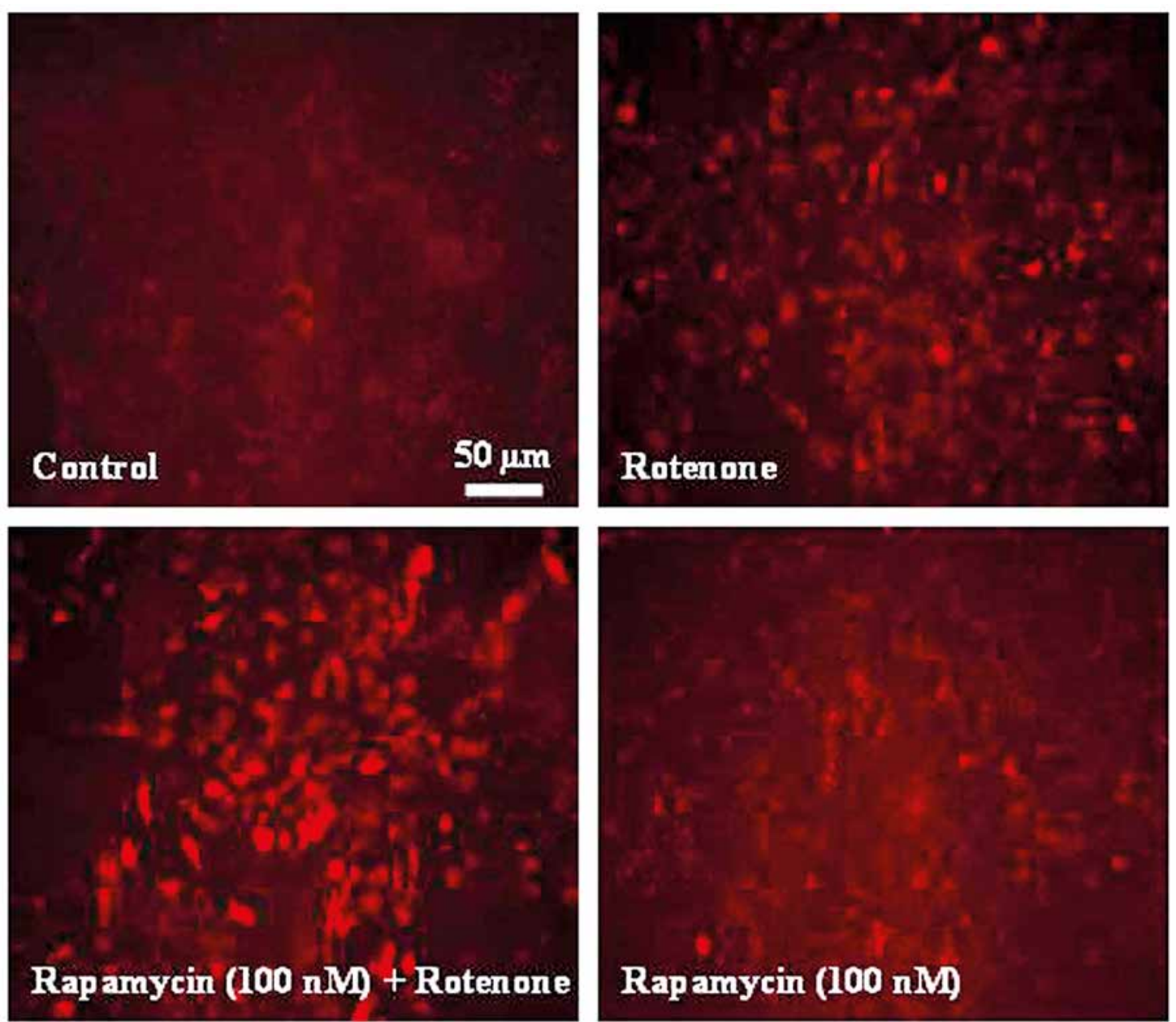

Fig. 4. A) LysoTracker Deep Red fluorescence intensity in primary mesencephalic cell cultures. $100 \%$ corresponds to the intensity of LysoTracker Deep Red in primary mesencephalic cell cultures after 12 DIV. Values represent the mean \pm SEM of three independent experiments with four wells in each treatment. Fluorescence intensity was determined densitometrically from 24 randomly selected micrographs in each experiment (6 photos from each well) ( $\left.{ }^{\#} p<0.01,{ }^{*} p<0.001\right)$. B) Representative micrographs showing that treatment of cultures with rapamycin increased LysoTracker Deep Red fluorescence intensity compared to rotenone-treated cultures. 


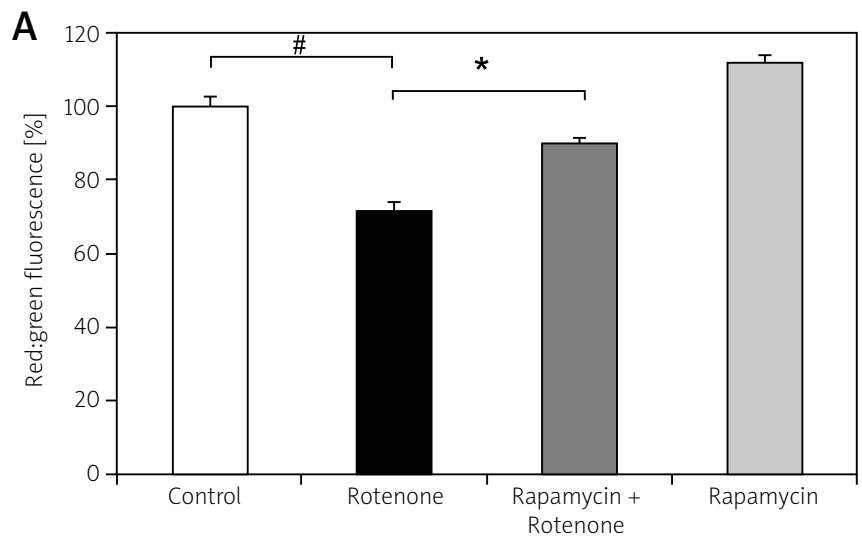

B
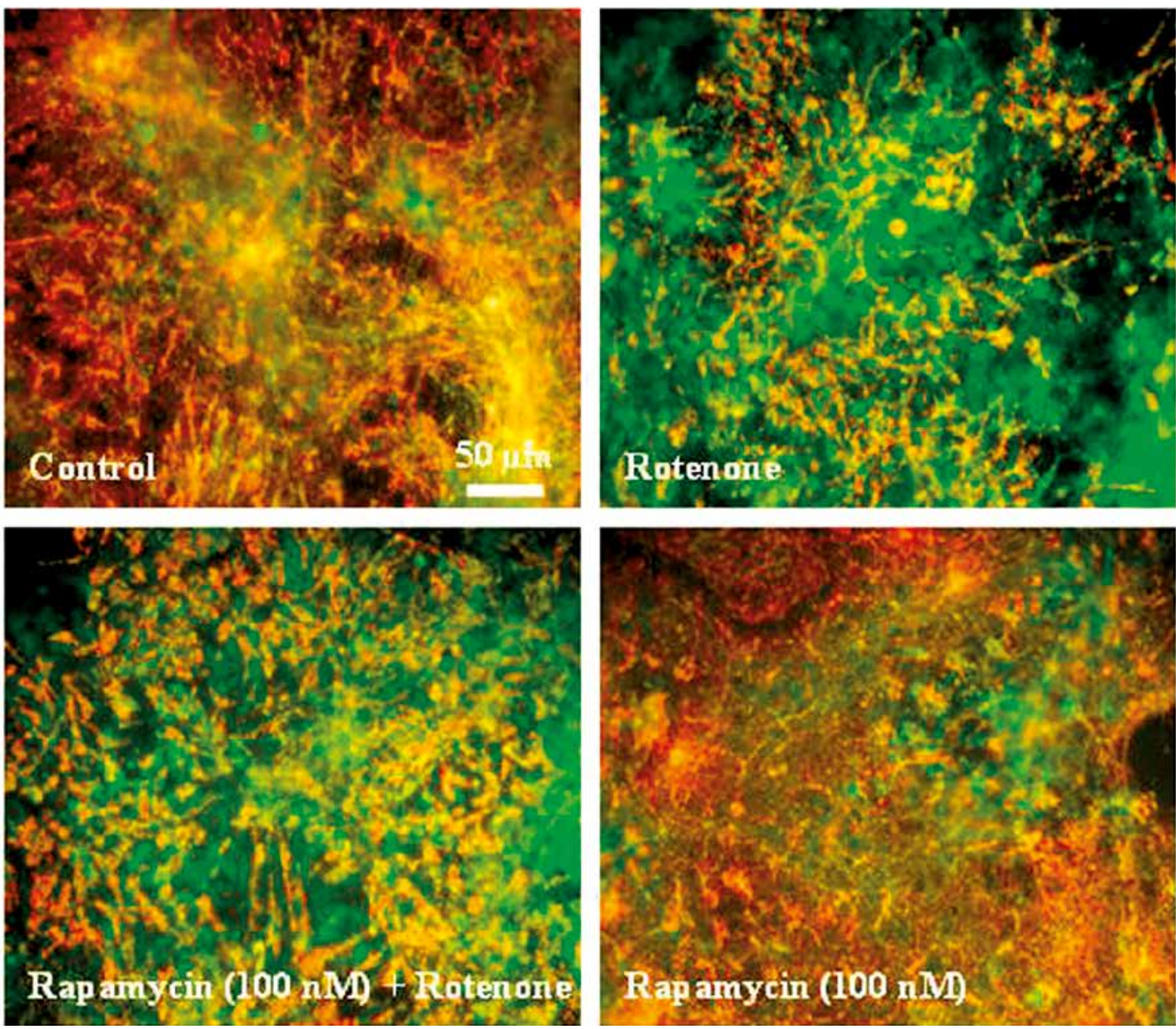

Fig. 5. A) Red:green fluorescence ratio of JC-1 in primary mesencephalic cell cultures. $100 \%$ corresponds to the red:green fluorescence ratio of JC-1 in primary mesencephalic cell cultures after 12 DIV. Values represent the mean \pm SEM of three independent experiments with four wells in each treatment. Red:green fluorescence ratio of JC-1 was determined densitometrically from 24 randomly selected micrographs in each experiment (6 photos from each well) ( $\left.{ }^{\#} p<0.001,{ }^{*} p<0.001\right)$. (B) Representative micrographs showing that treatment of cultures with rapamycin increased red fluorescence compared to rotenone-treated cultures which exhibit marked green fluorescence. 
B
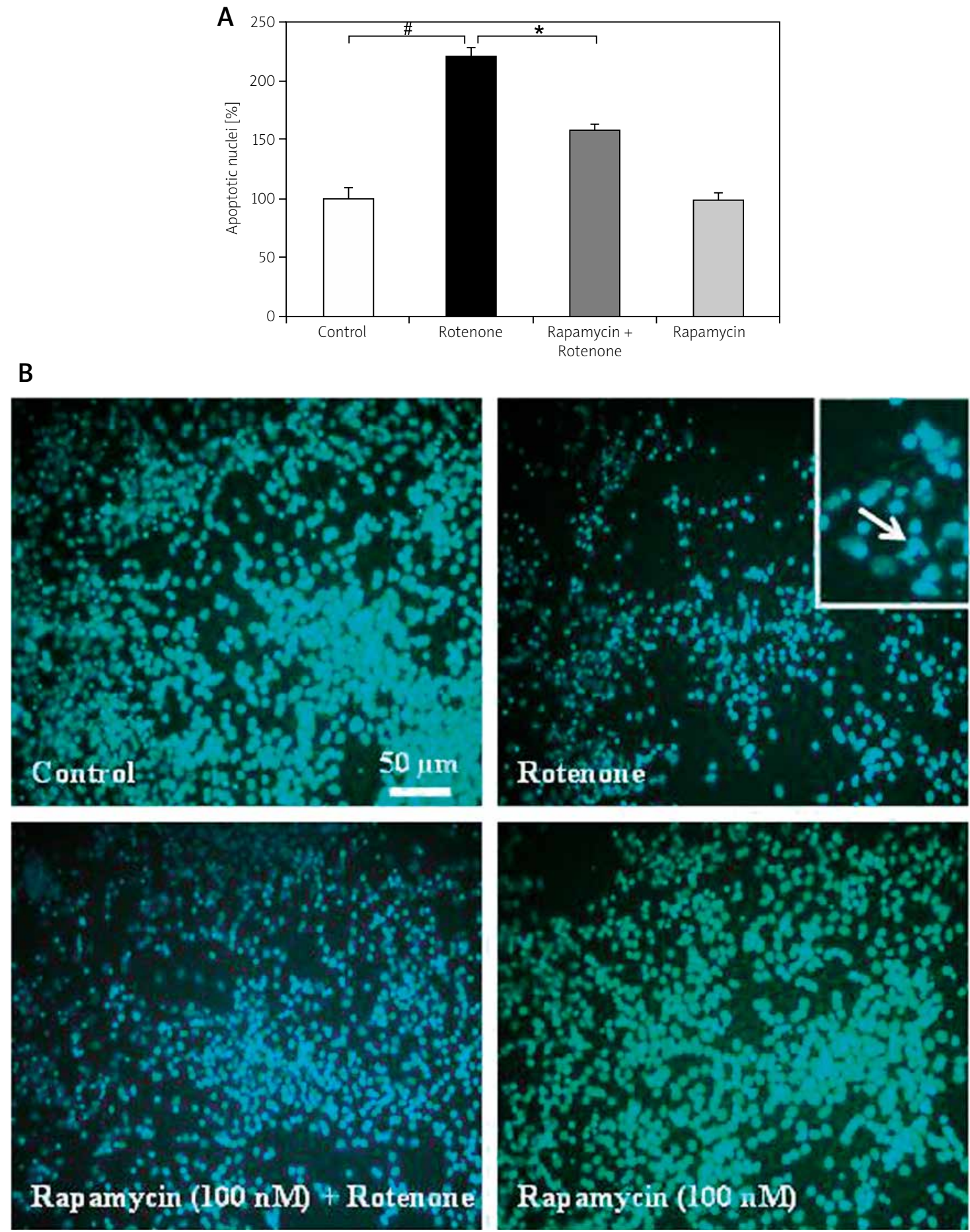

Fig. 6. (A) Number of nuclei showing apoptotic features with condensed and fragmented chromatin in primary mesencephalic cell cultures. $100 \%$ corresponds to the number of apoptotic nuclei (the average number of apoptotic nuclei was 38 nuclei/photo) in untreated control cultures after 12 DIV. Values represent the mean \pm SEM of three independent experiments with four wells in each treatment. Twenty-four photos were taken from each experiment ( 6 photos from each well) $\left.{ }^{\#} p<0.001,{ }^{*} p<0.001\right)$. B) Representative micrographs showing that treatment of cultures with rapamycin decreased the number of apoptotic nuclei compared to rotenone-treated cultures. Insert shows apoptotic nuclei at $20 \times$ magnification. 
nuclei by about $100 \%$ compared to rotenone-treated cultures (Fig. 6A). Apoptotic nuclei showed highly condensed and fragmented chromatin (Fig. 6B). Rapamycin alone did not affect the number of apoptotic nuclei compared to untreated control culture (Fig. 6A,B).

\section{Discussion}

In the present study, rotenone-treated primary mesencephalic cell culture was used as a neurotoxicity model to investigate the neuroprotective potential of the autophagy inducer rapamycin on dopaminergic neurons relevant to PD. In summary, rotenone was shown to 1) decrease the survival of dopaminergic neurons; 2) increase the release of $\mathrm{LDH}$ into the culture medium; 3) disrupt the mitochondrial $\Delta \psi m$ of cultured cells and 4) increase the features of apoptotic cell death in primary mesencephalic cell culture. This neurotoxic effect of rotenone has been reported in different in vitro and in vivo models since Betarbet and her colleagues [3] described rotenone as a PD neurotoxin in 2000. For instance, Radad et al. [31] found that exposure of primary mesencephalic cell culture to $20 \mathrm{nM}$ rotenone destroyed dopaminergic neurons and resulted in a higher level of LDH in the culture medium. Im et al. [15] reported that rotenone decreased the viability and survival of PC12 cells. Tapias et al. [37] described a loss of dopamine neurons and nigrostriatal terminals as a result of rotenone treatment in rats. Using JC-1 and DAPI fluorescence dyes we showed that rotenone decreased the $\Delta \psi \mathrm{m}$ and induced apoptotic cell death in our mesencephalic cell cultures, as indicated by the decreased red:green fluorescence ratio of JC-1 and increased number of nuclei with condensed and fragmented chromatin, respectively. Similarly, Hu et al. [14] demonstrated that rotenone caused a loss of $\Delta \psi \mathrm{m}$ and induced apoptotic cell death in SH-SY5Y cells. Mitochondrial damage by rotenone played a central role in apoptotic cell death through interrupting cellular energy metabolism, increasing ROS production and the release of apoptotic factors into the cytosol [2].

Our results showed that rapamycin rescued a significant number of dopaminergic neurons and decreased the release of LDH into the culture medium when concomitantly added with rotenone to primary mesencephalic cell cultures. Likewise, similar neuroprotective effects of rapamycin have been reported in some in vitro and in vivo models of neurodegeneration. For example, Malagelada et al. [21] found that rapamycin protected $\mathrm{PC} 12$ cells from 6-OHDA toxicity. Jiang et al. [16] observed that rapamycin provided behavioral improvements and protected against the loss of dopaminergic neurons in a rat model of PD.

Rapamycin increased cellular fluorescence of LysoTracker Deep Red compared to rotenone-treated cultures, indicating that rapamycin upregulated an autophagic process in cultured cells. In line with our results, Chikte et al. [6] reported that the signals of LysoTracker Deep Red were increased as the result of rapamycin treatment and could be used as a marker for autophagy in Jurkat T-cell and K562 erythro-myeloid cell lines. He and Klionskey [12] also correlated the fluorescence signals of LysoTracker with the upregulation of autophagy in zebrafish. Staining of cultures co-administered with rapamycin and rotenone with JC-1 fluorescent dye showed that rapamycin enhanced $\Delta \psi \mathrm{m}$ as it increased the red:green fluorescence ratio of JC-1 compared to cultures treated with rotenone alone. Rapamycin was similarly reported to enhance $\Delta \psi \mathrm{m}$ in the human breast cancer cell line MCF-7, as shown by the JC-1 potentiometric dye [24]. Moreover, rapamycin was reported to ameliorate mitochondrial defects in cells from individuals with the PARK2 mutation through rescuing $\Delta \psi \mathrm{m}$ [35]. Counting of apoptotic nuclei using blue-fluorescent DAPI nucleic acid stain indicated that rapamycin decreased rotenone-induced apoptotic cell death in primary mesencephalic cell cultures. Yin et al. [41] and Jing et al. [17] similarly reported an antiapoptotic effect for rapamycin against transient focal cerebral ischemia/reperfusion and subarachnoid hemorrhage in mice and rats, respectively. The antiapoptotic effect of rapamycin was reported to be mediated by decreasing Bax production and the downstream release of cytochrome c from mitochondria to the cytosol [17]. In addition to aforementioned mechanisms of neuroprotection, rapamycin was found to protect neuronal cells through some other pathways. In this context, Tain et al. [35] found that activation of the translation inhibitor 4E-BP protected dopaminergic neurons in parkin and PINK1 mutant Drosophila. Jiang et al. [16] reported that reduction of oxidative stress is one of the underlying mechanisms that mediate neuroprotection in a 6-OHDA rat model of PD.

Taking all the data together, our study shows that rapamycin protected dopaminergic neurons against rotenone-induced cell death in primary mesencephalic cell culture. This neuroprotection might be attributed to increasing autophagy, enhancing mitochondrial 
membrane potential and decreasing apoptotic cell death in primary mesencephalic cell culture.

\section{Disclosure}

\section{The authors report no conflict of interest.}

\section{References}

1. Banerjee R, Beal MF, Thomas B. Autophagy in neurodegenerative disorders: pathogenic roles and therapeutic implications. Trends Neurosci 2010; 33: 541-549.

2. Beal MF. Energetics in the pathogenesis of neurodegenerative diseases. Trends Neurosci 2000; 23: 298-304.

3. Betarbet R, Sherer TB, Mackenzie G, Garcia-Osuna M, Panov AV Greenamyre JT. Chronic systemic pesticide exposure reproduces features of Parkinson's disease. Nat Neurosci 2000; 3: 1301-1306.

4. Boll MC, Alcaraz-Zubeldia M, Rios C. Medical management of Parkinson's disease: focus on neuroprotection. Curr Neuropharmacol 2011; 9: 350-359.

5. Carloni S, Buonocore G, Longini M, Proietti F, Balduini W. Inhibition of rapamycin-induced autophagy causes necrotic cell death associated with Bax/Bad mitochondrial translocation. Neuroscience 2012; 203: 160-169.

6. Chikte S, Panchal N, Warnes G. Use ofLysoTracker dyes: A flow cytometric study of autophagy. Cytometry A 2014; 85: 169-178.

7. Dehay B, Bové J, Rodríguez-Muela N, Perier C, Recasens A, Boya P, Vila M. Pathogenic lysosomal depletion in Parkinson's disease. J Neurosci 2010; 30: 12535-12544.

8. Douna H, Bavelaar BM, Pellikaan H, Olivier B. Neuroprotection in Parkinson's disease: A systemic review of the preclinical data. The Open Pharmacology Journal 2012; 6: 12-26.

9. Eriksen JL, Wszolek Z, Petrucelli L. Molecular pathogenesis of Parkinson disease. Arch Neurol 2005; 62: 353-357.

10. Gwag BJ, Lobner D, Koh JY, Wie MB, Choi DW. Blockade of glutamate receptors unmasks neuronal apoptosis after oxygen-glucose deprivation in vitro. Neuroscience 1995; 68: 615-619.

11. Hauser RA. Early pharmacologic treatment in Parkinson's disease. Am J Manag Care 2010; 16: 100-107.

12. He C, Klionsky DJ. Analyzing autophagy inzebrafish. Autophagy 2010; 6: 642-644

13. Hirsch EC. Future drug targets for Parkinson's disease. Bull Acad Natl Med 2012; 196: 1369-1377.

14. Hu LF, Lu M, Wu ZY, Wong PT, Bian JS. Hydrogen sulfide inhibits rotenone-induced apoptosis via preservation of mitochondrial function. Mol Pharmacol 2009; 75: 27-34.

15. Im AR, Kim YH, Uddin MR, Chae S, Lee HW, Kim YS, Lee MY. Neuroprotective effects of Lycium chinense Miller against rotenone-induced neurotoxicity in PC12 Cells. Am J Chin Med 2013; 41: 1343-1359.

16. Jiang J, Jiang J, Zuo Y, Gu Z. Rapamycin protects the mitochondria against oxidative stress and apoptosis in a rat model of Parkinson's disease. Int J Mol Med 2013; 31: 825-832.

17. Jing CH, Wang L, Liu PP, Wu C, Ruan D, Chen G. Autophagy activation is associated with neuroprotection against apoptosis via a mitochondrial pathway in a rat model of subarachnoid hemorrhage. Neuroscience 2012; 213: 144-153.
18. Kanno H, Ozawa H, Sekiguchi A, Yamaya S, Tateda S, Yahata K, Itoi $\mathrm{E}$. The role of mTOR signaling pathway in spinal cord injury. Cell Cycle 2012; 11: 3175-3179.

19. Koh JY, Choi DW. Quantitative determination of glutamate mediatedcortical neuronal injury in cell culture by lactate dehydrogenase efflux assay. J Neurosci Methods 1987; 20: 83-90.

20. Lipski J, Nistico R, Berretta N, Guatteo E, Bernardi G, Mercuri NB. L-DOPA: a scapegoat for accelerated neurodegeneration in Parkinson's disease? Prog Neurobiol 2011; 94: 389-407.

21. Malagelada C, Jin ZH, Jackson-Lewis V, Przedborski S, Greene LA. Rapamycin protects against neuron death in in vitro and in vivo models of Parkinson's disease. J Neurosci 2010; 30: 1166-11675.

22. Meinel J, Radad R, Rausch WD, Reichman H, Gille G. Cabergoline protects dopaminergic neurons against rotenone-induced cell death in primary mesencephalic cell culture. Folia Neuropathologica 2015; 53: 29-40.

23. Oster S, Radad K, Scheller D, Hesse M, Balanzew W, Reichmann H, Gille G. Rotigotine protects against glutamate toxicity in primary dopaminergic cell culture. Eur J Pharmacol 2014; 724: 31-42.

24. Parganlija D, Klinkenberg M, Domínguez-Bautista J, Hetzel M, Gispert S, Chimi MA, Dröse S, Mai S, Brandt U, Auburger G, Jendrach M. Loss of PINK1 Impairs Stress-Induced Autophagy and Cell Survival. PLoS One 2014; 21: e95288.

25. Park HJ, Shin JY, Kim HN, Oh SH, Lee PH. Neuroprotective effects of mesenchymal stem cells through autophagy modulation in a parkinsonian model. Neurobiol Aging 2014; 35: 1920-1928.

26. Park JH, Lee JE, Lee SJ, Park SJ, Park KH, Jeong M, Koh HC. Potential autophagy enhancers protect against fipronil-induced apoptosis in SH-SY5Y cells. Toxicol Lett 2013; 223: 25-34.

27. Park SS, Lee D. Selective loss of dopaminergic neurons and formation of Lewy body-like aggregations in alpha-synuclein transgenic fly neuronal cultures. Eur J Neurosci 2006; 23: 29082914.

28. Prakash KM, Tan EK. Development of Parkinson's disease biomarkers. Expert Rev Neurother 2010; 10: 1811-1825.

29. Pyszko JA, Strosznajder JB. The key role of sphingosine kinases in the molecular mechanism of neuronal cell survival and death in an experimental model of Parkinson's disease. Folia Neuropathol 2014; 52: 260-269.

30. Radad KS, Al-Shraim MM, Moustafa MF, Rausch WD. Neuroprotective role of thymoquinone against 1-methyl-4-phenylpyridinium-induced dopaminergic cell death in primary mesencephalic cell culture. Neurosciences (Riyadh) 2015; 20: 10-16.

31. Radad K, Gille G, Rausch WD. Dopaminergic neurons are preferentially sensitive to long-term rotenone toxicity in primary cell culture. Toxicol In Vitro 2008; 22: 68-74.

32. Radad K, Scheller D, Rausch WD, Reichmann H, Gille G. Neuroprotective effect of rotigotine against complex I inhibitors, $\mathrm{MPP}+$ and rotenone, in primary mesencephalic cell culture. Folia Neuropathol 2014; 52: 179-186.

33. Seto B. Rapamycin and mTOR: a serendipitous discovery and implications for breast cancer. Clin Transl Med 2012; 1: 29.

34. Singer C. Managing the patient with newly diagnosed Parkinson disease. Cleve Clin J Med 2012; 9: 3-7. 
35. Tain LS, Mortiboys H, Tao RN, Ziviani E, Bandmann O, Whitworth AJ.Rapamycin activation of $4 \mathrm{E}-\mathrm{BP}$ prevents parkinsonian dopaminergic neuron loss. Nat Neurosci 2009; 12: 1129-1135.

36. Tang P, Hou H, Zhang L, Lan X, Mao Z, Liu D, He C, Du H, Zhang L. Autophagy reduces neuronal damage and promotes locomotorrecovery via inhibition of apoptosis after spinal cord injury in rats. Mol Neurobiol 2014; 49: 276-287.

37. Tapias V, Cannon JR, Greenamyre JT. Pomegranate juice exacerbates oxidative stress and nigrostriatal degeneration in Parkinson's disease. Neurobiol Aging 2014; 35: 1169-1176.

38. Vézina C, Kudelski A, Sehgal SN. Rapamycin (AY-22,989), a new antifungal antibiotic. I. Taxonomy of the producing streptomycete and isolation of the active principle. J Antibiot (Tokyo) 1975; 28: 721-726.

39. White RJ, Reynolds IJ. Mitochondrial depolarization in glutamate-stimulated neurons: an early signal specific to excitotoxin exposure. J Neurosci 1996; 16: 5688-5697.

40. Worth PF. How to treat Parkinson's disease in 2013. Clin Med 2013; 13: 93-96.

41. Yin L, Ye S, Chen Z, Zeng Y. Rapamycin preconditioning attenuates transient focal cerebral ischemia/reperfusion injury in mice. Int J Neurosci 2012; 122: 748-756. 